\title{
A General Conductivity Expression for Space-Charge-Limited Conduction in Ferroelectrics and Other Solid Dielectrics
}

\author{
Ho-Kei Chan ${ }^{1,2}$ \\ ${ }^{1}$ School of Physics, Trinity College Dublin, College Green, Dublin 2, \\ 2Department of Applied Physics, Hong Kong Polytechnic University, Kowloon, \\ Ireland \\ ${ }^{2}$ Hong Kong, China
}

\section{Introduction}

For a current density $J$ and an applied voltage $V$, the experimentally observable MottGurney law $J \sim V^{2}$ (Carbone et al., 2005; Coelho, 1979; Laha \& Krupanidhi, 2002; Pope \& Swemberg, 1998; Suh et al., 2000) for steady-state trap-free space-charge-limited conduction (SCLC) inside a plane-parallel dielectric capacitor is derived from three independent assumptions - (i) the presence of a single free-carrier type (i.e. injection of only $p$-type or $n$ type free charge-carriers into the dielectric), (ii) the absence of an intrinsic (Ohmic) conductivity, and (iii) the existence of a constant dielectric permittivity $\varepsilon$ (i.e. the material being a linear dielectric). Due to the limited applicability of the Mott-Gurney law, there has been the need to derive a more general SCLC formula that applies to cases (i) with the simultaneous presence of $p$-type and $n$-type free charge-carriers, (ii) with the presence of a small but finite intrinsic conductivity $\sigma_{i n}$, as well as (iii) with any possible field dependence of the dielectric permittivity $\varepsilon$. In 2003, while we were searching for a theoretical explanation to the well-known experimental observation of polarization offsets (Schubring et al., 1992) in compositionally graded ferroelectric films, we employed the law of mass action to derive a general local conductivity expression for double-carrier-type SCLC in solid dielectrics and showed numerically that SCLC is a possible origin of the observation of polarization offsets in those graded ferroelectric films (Chan et al., 2004). In those graded films, there exist gradients of electric displacements and therefore, according to Gauss' law, a corresponding presence of free space-charge. The local electrical conductivity is influenced, or even limited, by the presence of free space-charge and consequently becomes non-Ohmic. An important conclusion from this general local conductivity expression is the necessary dominance of a single free-carrier type at the limit of zero intrinsic conductivity, which links two of the three independent assumptions in the original derivation of the Mott-Gurney law. In a later study (Zhou et al., 2005a), we have employed this new conductivity expression to understand the origin of imprint effect in homogeneous ferroelectric films. In this Book Chapter, we will (i) review the original derivation of the Mott-Gurney law (Coelho, 1979; Pope \& Swemberg,

e-mail: epkeiyeah@yahoo.com.hk 
1998), (ii) review the original problem of polarization offsets in compositionally graded ferroelectric films (Schubring et al., 1992) and explain why such a new local conductivity expression was needed in our theoretical investigation, (iii) review our original derivation of this general local conductivity expression (Chan et al., 2004), (iv) review our study on the limiting case of zero intrinsic conductivity (Chan et al., 2007; Zhou et al., 2005a), (v) supply the derivation of a general expression, via the mass-action approximation, for the corresponding local diffusion-current density (Chan, unpublished), which incorporates the Einstein relations and takes into account the possible presence of a temperature gradient, (vi) present an alternative derivation of this local conductivity expression (Chan, unpublished) with a detailed theoretical justification of the mass-action approximation, and (vii) discuss, in the concluding section, possible future work as to a better understanding of the mechanism of charge flow in a compositionally graded ferroelectric film as well as of the scope of applicability of the general local conductivity expression.

\section{Derivation of the Mott-Gurney law}

Space-charge-limited conduction (SCLC) in a solid dielectric occurs when free chargecarriers are injected into the dielectric sample at relatively large electric fields (Carbone et al., 2005; Coelho, 1979; Laha \& Krupanidhi, 2002; Pope \& Swemberg, 1998; Suh et al., 2000). For the case of SCLC in a trap-free plane-parallel dielectric capacitor (Fig. 1), the current density $J$ and the applied voltage $V$ follow a scaling relation

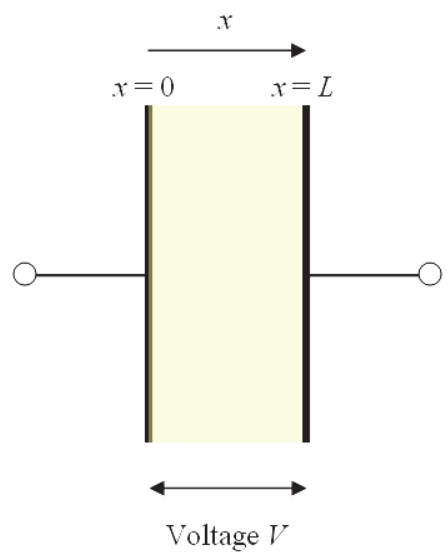

Fig. 1. Schematic diagram of a plane-parallel dielectric capacitor. $V$ is the applied voltage, and $x$ is the position from the electrode where the electric current flows into the dielectric sample. The positive direction of $x$ is the flow direction of the electric current.

$$
J \sim V^{2}
$$

which can be observed experimentally (Carbone et al., 2005; Laha \& Krupanidhi, 2002; Suh et al., 2000), and can be derived theoretically (Coelho, 1979) as follows: Consider the case of SCLC by only $p$-type free charge-carriers, and let this case be denoted by a subscript $p$ in each of the quantities involved. Let the charge mobility $\mu_{p}>0$ and the dielectric permittivity 
$\varepsilon>0$ be both independent of the position $x$ from the electrode where the electric current flows into the dielectric sample. The flow direction of the electric current is then given by the positive direction of $x$. According to Gauss' law, the free-charge density is given by

$$
\rho_{p}(x)=\varepsilon \frac{d E_{p}(x)}{d x} \geq 0
$$

where $E_{p}(x)$ is the electric field along the positive direction of $x$. At steady states, the continuity equation

$$
\vec{\nabla} \cdot \vec{J}_{p}=0
$$

implies that the current density $J_{p}$ is independent of the position $x$. At the absence of both intrinsic (Ohmic) conductivity and diffusion currents, this current density is given by

$$
J_{p}=\mu_{p} \rho_{p}(x) E_{p}(x)=\frac{\mu_{p} \varepsilon}{2} \frac{d E_{p}(x)^{2}}{d x}
$$

A spatial integration of Eq. (4) from 0 to $x$ using the boundary condition $E_{p}(0)=0$ yields

$$
J_{p}=\frac{\mu_{p} \varepsilon}{2 x} E_{p}(x)^{2} \geq 0
$$

or equivalently

$$
E_{p}(x)=\sqrt{\frac{2 J_{p} x}{\mu_{p} \varepsilon}} \geq 0
$$

The present justification for the boundary condition $E_{p}(0)=0$ is that, at the boundary $x=$ 0 , any electric field along the positive direction of $x$, if it ever exists, would be 'neutralized' by an electric field in the opposite direction as generated by the injected $p$ type free carriers (Coelho, 1979). Here we provide a "quantitative explanation" for this: At $x=0$, the electric field satisfies the inequality $E_{p}(0) \geq 0$. But since, at this boundary, there is a step change of the free-charge density from zero to some finite positive value, the inequality $E_{p}(0) \leq 0$ is also satisfied as any finite local electric field is also expected to be pointing along the negative direction of $x$. The simultaneous inequalities $E_{p}(0) \geq 0$ and $E_{p}(0) \leq 0$ together imply $E_{p}(0)=0$. Using Kirchoff's loop law (i.e. Kirchoff's voltage law) and Eq. (6), we obtain

$$
V=\int_{0}^{L} E_{p}(x) d x=\left(\frac{8 J_{p}}{9 \mu_{p} \varepsilon}\right)^{1 / 2} L^{3 / 2}
$$

Hence,

$$
J_{p}=\left(\frac{9 \mu_{p} \varepsilon}{8 L^{3}}\right) V^{2} \sim V^{2}
$$


If $n$-type instead of $p$-type free carriers are injected into the dielectric sample, Eqs. (2) and (4) should be replaced by

$$
\rho_{n}(x)=\varepsilon \frac{d E_{n}(x)}{d x} \leq 0
$$

and

$$
J_{n}=-\mu_{n} \rho_{n}(x) E_{n}(x)=-\frac{\mu_{n} \varepsilon}{2} \frac{d E_{n}(x)^{2}}{d x}
$$

respectively, where $\mu_{n}>0$, and the subscript $n$ denotes the case of SCLC by only $n$-type free carriers. Following a derivation similar to that for the case of $p$-type SCLC but with a consideration of the boundary condition $E_{n}(L)=0$, we obtain

$$
J_{n}=\left(\frac{9 \mu_{n} \varepsilon}{8 L^{3}}\right) V^{2} \sim V^{2}
$$

It follows that the scaling relation $J \sim V^{2}$ holds regardless of whether the injected free carriers are $p$-type or $n$-type.

\section{Polarization offsets in graded ferroelectrics and the need for a general local conductivity expression}

Our theoretical investigation of SCLC began with a study of the well-known phenomenon of polarization offsets in compositionally graded ferroelectric films (Bao et al. 2000a, 2000b, 2000c, 2001a, 2001b; Bouregba et al., 2003; Brazier et al., 1998; Chen et al., 1999; Mantese et al., 1997; Matsuzaki \& Funakubo, 1999; Schubring et al., 1992). Ferroelectric materials are materials that generally exhibit nonlinear, hysteretic $D-E$ or $P-E$ relations, where $D$ is the electric displacement, $P$ is the polarization and $E$ is the electric field. In contrast to the "normal" hysteresis loops, i.e. loops that are centred at the origin of the $D-E$ or $P-E$ plot, as observed for homogeneous ferroelectric films, it was first reported in Physical Review Letters (Schubring et al., 1992) that compositionally graded ferroelectric films can exhibit vertical displacements of their hysteresis loops along the polarization axis, i.e. polarization offsets, when driven by an alternating applied electric field and placed inside a Sawyer-Tower circuit (Chan et al., 2004; Sawyer \& Tower, 1930) for typical D-E measurements of ferroelectric materials. This intriguing phenomenon, believed to have potential device applications in infrared detection, actuation and energy storage (Mantese \& Alpay, 2005), has triggered much subsequent research in the ferroelectrics community into a theoretical understanding of its origin (Alpay et al., 2003; Bouregba et al., 2003; Brazier et al., 1999; Chan et al., 2004; Fellberg et al., 2001; Mantese \& Alpay, 2005; Mantese et al., 1997; Okatan et al., 2010; Poullain et al., 2002).

Such a phenomenon of polarization offsets in compositionally graded ferroelectric films is characterized by three key experimental observations: (i) The shift magnitude exhibits a strong dependence on electric field and temperature (Schubring et al., 1992). In particular, it typically exhibits a power 3 to 5 dependence on the magnitude of the applied electric field (Bao et al., 2000a, 2000b, 2000c, 2001b; Bouregba et al., 2003; Brazier et al., 1998; Mantese et al., 1997); (ii) The vertical hysteresis shift changes from upward to downward, or vice versa, 
when the compositional gradient is inverted (Bouregba et al., 2003; Brazier et al., 1998; Mantese et al., 1997). (iii) A vertical polarization offset typically develops like the charging up of a capacitor, where the "time constant" is of the order of magnitude as the product of the capacitance and input impedance of the reference capacitor in the Sawyer-Tower circuit. In the past two decades, various theoretical ideas and models have been proposed to account for the origin of this phenomenon: Originally, the vertical hysteresis shift, or "polarization offset", was thought of as a static polarization developed across the graded ferroelectric film (Mantese et al., 1997), upon excitation by an alternating applied electric field. However, the experimental values of those "offsets" are at least an order of magnitude larger than the typical spontaneous polarization of the ferroelectric material, and therefore such a large static polarization component is deemed unlikely, if not impossible (Brazier et al., 1999). Other theoretical considerations include an interpretation of the vertical hysteresis shift as the result of a static voltage developed across the ferroelectric film (Brazier et al., 1999), as a result of asymmetric electrical conduction by leakage currents in the film (Bouregba et al., 2003), and as an effect of space charge in a perfectly insulating ferroelectric film (Okatan et al., 2010).

In our theoretical investigation where all of the key experimental observations mentioned above were reproduced theoretically (Chan et al., 2004), we proposed that the observation of polarization offsets in a compositionally graded ferroelectric film is a result of "timedependent" space-charge-limited conduction inside the graded film, where the term "timedependent" was used because the ferroelectric film was excited by an alternating applied electric field, in contrast to the abovementioned case of steady-state SCLC, in the absence of displacement currents, as described by the Mott-Gurney law. This central assumption was based on the following considerations: A similar phenomenon of polarization offsets was observed for homogeneous ferroelectric films in the presence of a temperature gradient (Fellberg et al., 2001), where the vertical hysteresis shift disappeared when the applied temperature gradient was removed. This suggests that, for this particular case, the shift is a result of thermally induced gradients in the polarization $P$ (Alpay et al., 2003; Fellberg et al., 2001), or more generally in the electric displacement $D$, where the latter implies the presence of free space-charge according to Gauss' law. If the vertical hysteresis shifts that arise from compositional and thermal gradients are of the same origin, the observation of polarization offsets in compositionally graded ferroelectric films might be mainly a result of electrical conduction by free space-charge, i.e. SCLC. Here, we have deliberately aborted the assumption of a perfectly insulating ferroelectric film, as adopted by some other schools of thought (Alpay et al., 2003; Mantese \& Alpay, 2005; Okatan et al., 2010), because experimental observations of small but finite leakage currents, as well as of SCLC currents, have been widely reported in the literature for ferroelectrics and other solid dielectrics (Bouregba et al., 2003; Carbone et al., 2005; Coelho, 1979; Laha \& Krupanidhi, 2002; Pope \& Swemberg, 1998; Poullain et al., 2002; Suh et al., 2000) and they form a subject of their own (Pope \& Swemberg, 1998).

For our consideration of SCLC in compositionally graded ferroelectric films, the MottGurney law $J \sim V^{2}$ does not apply, due to the following reasons: (i) In the case of a timevarying applied voltage, there is also the presence of displacement currents so that the conduction current $J$, which now does not necessarily equal the total current, could be varying with position, i.e. Eq. (3) no longer holds. (ii) In many ferroelectric and dielectric materials, there exist two opposite types of free charge-carriers, $p$-type and $n$-type, with 
the system behaving like a wide bandgap semiconductor. Such double-carrier cases are not considered by the Mott-Gurney law. (iii) The Mott-Gurney law offers no way to take into account any small but finite intrinsic conductivity of a ferroelectric or dielectric material. (iv) For non-linear dielectric materials, the dielectric permittivity $\varepsilon$ is not a constant, usually field-dependent, and for ferroelectrics it is even time- or historydependent. And for inhomogeneous materials, e.g. compositionally graded ferroelectrics, $\varepsilon$ typically exhibits a spatial variation. In view of these limitations, we have derived a new local conductivity expression (Chan et al., 2004) that is applicable to cases with the presence of both $p$-type and $n$-type free carriers, with a small but finite local conductivity, and with any possible dependence of the local dielectric permittivity $\varepsilon$ on the system parameters. It is worth pointing out that a corresponding global conductivity expression, e.g. the Mott-Gurney law, must be obtained via the continuity condition in Eq. (3) and can only be derived with knowledge of the relation of $\varepsilon$ with other system parameters. For example, in the derivation of the Mott-Gurney law, $\varepsilon$ is a known quantity - it is taken as a constant, independent of any system parameter. In the following Section, we will review our original derivation (Chan et al., 2004) of this local conductivity expression, where the law of mass action was employed as an ad hoc approximation to describe the interactions between the two opposite types of free charge-carriers. A detailed justification of this mass-action approximation is provided in subsequent Sections.

\section{Original derivation of the general local conductivity expression}

For a time-varying applied voltage $V(t)$, the local conduction-current density $J_{c}(x, t)$, the local electric field $E(x, t)$ as well as the local electric displacement $D(x, t)$ are generally functions of the position $x$ and the time $t$. The local conduction-current density is related to the local electric field via a generally time-dependent local conductivity $\sigma(x, t)$ :

$$
J_{c}(x, t)=\sigma(x, t) E(x, t)
$$

Consider the simultaneous presence of $p$-type and $n$-type free charge-carriers with the position-dependent mobilities $\mu_{p}(x)$ and $-\mu_{n}(x)$, and of electric charges $q$ and $-q$, respectively. Here we have chosen $\mu_{p}(x), \mu_{n}(x)$ and $q$ to all be positive quantities. Due to charge neutrality, the intrinsic number concentrations of the two opposite types of free carriers are equal, and they are here both denoted by the symbol $C_{i n}(x)$. The time-dependent conductivity can then be expressed as

$$
\begin{aligned}
\sigma(x, t)=q \mu_{p}(x)\left[C_{i n}(x)+\Delta p(x, t)\right] \\
+[-q]\left[-\mu_{n}(x)\right]\left[C_{i n}(x)+\Delta n(x, t)\right]
\end{aligned}
$$

where $\Delta p(x, t)$ and $\Delta n(x, t)$ are respectively the differences between the intrinsic and total number concentrations for each type of free carriers. Here we define a generally positiondependent local intrinsic conductivity

$$
\sigma_{\text {in }}(x) \equiv q\left[\mu_{p}(x)+\mu_{n}(x)\right] C_{i n}(x)
$$

such that the time-dependent conductivity in Eq. (13) can be expressed as

$$
\sigma(x, t)=\sigma_{i n}(x)+q\left[\mu_{p}(x) \Delta p(x, t)+\mu_{n}(x) \Delta n(x, t)\right]
$$


According to Gauss' law, the density of free space-charge is given by

$$
\begin{aligned}
\frac{\partial D(x, t)}{\partial x}= & q\left[C_{i n}(x)+\Delta p(x, t)\right]+[-q]\left[C_{i n}(x)+\Delta n(x, t)\right] \\
& =q[\Delta p(x, t)-\Delta n(x, t)]
\end{aligned}
$$

Since there generally exist interactions, i.e. generation and recombination, between the two opposite types of free charge-carriers, the free-carrier concentrations $C_{i n}(x)+\Delta p(x, t)$ and $C_{i n}(x)+\Delta n(x, t)$ are not independent of each other, and should be described by an additional physical constraint

$$
F[\Delta p(x, t), \Delta n(x, t)]=0
$$

As an ah hoc approximation, we regard any ferroelectric or dielectric material as a wide bandgap semiconductor and then employ the equilibrium law of mass action to be this additional physical constraint:

$$
C_{i n}(x)^{2}=\left[C_{i n}(x)+\Delta p(x, t)\right]\left[C_{i n}(x)+\Delta n(x, t)\right]
$$

The physics described by Eq. (18) is as follows (Chan et al., 2007): In a wide bandgap semiconductor, there are the endothermic generation and exothermic recombination of free charge-carriers. If the energy released by free-carrier recombination is immediately used for free-carrier generation, i.e. a "heat balance" condition, the rates of these endothermic and exothermic processes must be equal, and there would not be any net production of heat nor any net generation of free carriers. On the other hand, the rate of each of these processes can be assumed, as a first approximation, to be proportional to the product of the reactants' concentrations, i.e. the number of ways per unit volume the different types of reactants can combine to undergo the process. Since, for a dielectric insulator, the concentration of the source particles for free-carrier generation (e.g. valence electrons or molecules) is by definition orders of magnitude larger than the concentrations of the free carriers, the concentration of source particles, and hence the rate of free-carrier generation, should only have an insignificant time fluctuation and should practically be a material-pertaining property. It follows that any rate of free-carrier recombination, which we have here assumed to be proportional to the product of the concentrations of $p$-type and $n$-type free carriers, can all be taken as equal to the same approximately timeinvariant rate of free-carrier generation, hence Eq. (18).

Upon rewriting Eqs. (16) and (18) as

$$
\Delta p(x, t)=\frac{1}{q} \frac{\partial D(x, t)}{\partial x}+\Delta n(x, t)
$$

and

$$
C_{i n}(x)[\Delta p(x, t)+\Delta n(x, t)]+\Delta p(x, t) \Delta n(x, t)=0
$$

respectively, it can be seen that two quadratic equations, for $\Delta p(x, t)$ and $\Delta n(x, t)$ respectively, can be obtained: 


$$
\Delta p(x, t)^{2}+B_{p}(x, t) \Delta p(x, t)+C_{p}(x, t)=0
$$

and

$$
\Delta n(x, t)^{2}+B_{n}(x, t) \Delta n(x, t)+C_{n}(x, t)=0
$$

where

$$
\begin{gathered}
B_{p}(x, t) \equiv-\frac{1}{q} \frac{\partial D(x, t)}{\partial x}+2 C_{i n}(x) \\
C_{p}(x, t) \equiv-\frac{C_{i n}(x)}{q} \frac{\partial D(x, t)}{\partial x} \\
B_{n}(x, t) \equiv \frac{1}{q} \frac{\partial D(x, t)}{\partial x}+2 C_{i n}(x)
\end{gathered}
$$

and

$$
C_{n}(x, t) \equiv \frac{C_{i n}(x)}{q} \frac{\partial D(x, t)}{\partial x}
$$

It follows that

$$
\begin{aligned}
& q\left[\mu_{p}(x) \Delta p(x, t)+\mu_{n}(x) \Delta n(x, t)\right] \\
& =\frac{q}{2}\left\{\mu_{p}(x)\left[-B_{p}(x, t) \pm \sqrt{B_{p}(x, t)^{2}-4 C_{p}(x, t)}\right]\right. \\
& \left.\quad+\mu_{n}(x)\left[-B_{n}(x, t) \pm \sqrt{B_{n}(x, t)^{2}-4 C_{n}(x, t)}\right]\right\}
\end{aligned}
$$

Using the definitions of the quadratic coefficients $B_{p}(x, t), C_{p}(x, t), B_{n}(x, t)$ and $C_{n}(x, t)$, as well as the definition of $\sigma_{i n}(x)$ in Eq. (14), the following relations can be obtained:

$$
\begin{aligned}
-\frac{q}{2}[ & \left.\mu_{p}(x) B_{p}(x, t)+\mu_{n}(x) B_{n}(x, t)\right] \\
& =\frac{\left[\mu_{p}(x)-\mu_{n}(x)\right]}{2} \frac{\partial D(x, t)}{\partial x}-\sigma_{i n}(x)
\end{aligned}
$$

and

$$
\begin{gathered}
\frac{q}{2} \sqrt{B_{p}(x, t)^{2}-4 C_{p}(x, t)}=\frac{q}{2} \sqrt{B_{n}(x, t)^{2}-4 C_{n}(x, t)} \\
=\frac{1}{2} \sqrt{\left[\frac{\partial D(x, t)}{\partial x}\right]^{2}+\frac{4 \sigma_{i n}(x)^{2}}{\left[\mu_{p}(x)+\mu_{n}(x)\right]^{2}}}
\end{gathered}
$$

Using Eqs. (15), (28) and (29), Eq. (27) can be rewritten as 


$$
\begin{aligned}
& q\left[\mu_{p}(x) \Delta p(x, t)+\mu_{n}(x) \Delta n(x, t)\right] \\
& =\frac{\left[\mu_{p}(x)-\mu_{n}(x)\right]}{2} \frac{\partial D(x, t)}{\partial x}-\sigma_{i n}(x) \\
& +\frac{\left[ \pm \mu_{p}(x) \pm \mu_{n}(x)\right]}{2} \sqrt{\left[\frac{\partial D(x, t)}{\partial x}\right]^{2}+\frac{4 \sigma_{i n}(x)^{2}}{\left[\mu_{p}(x)+\mu_{n}(x)\right]^{2}}} \\
& =\sigma(x, t)-\sigma_{i n}(x)
\end{aligned}
$$

so that an expression for the time-dependent local conductivity $\sigma(x, t)$ can be obtained:

$$
\begin{aligned}
& \sigma(x, t)=\frac{\left[\mu_{p}(x)-\mu_{n}(x)\right]}{2} \frac{\partial D(x, t)}{\partial x} \\
& +\sqrt{\left\{\begin{array}{c}
\left.\frac{\left[ \pm \mu_{p}(x) \pm \mu_{n}(x)\right]}{2} \frac{\partial D(x, t)}{\partial x}\right\}^{2} \\
+\frac{\left[ \pm \mu_{p}(x) \pm \mu_{n}(x)\right]^{2} \sigma_{i n}(x)^{2}}{\left[\mu_{p}(x)+\mu_{n}(x)\right]^{2}}
\end{array}\right.}
\end{aligned}
$$

According to Eqs. (15), (16) and (31), for the case of $\Delta p(x, t)=\Delta n(x, t)=0$, we have

$$
\frac{\partial D(x, t)}{\partial x}=0
$$

and

$$
\sigma(x, t)=\sigma_{i n}(x)=\frac{\left[ \pm \mu_{p}(x) \pm \mu_{n}(x)\right]}{\left[\mu_{p}(x)+\mu_{n}(x)\right]} \sigma_{\text {in }}(x)
$$

where the latter implies that only the root of all upper positive signs in Eq. (31) is valid. Therefore, the correct expression for the time-dependent conductivity should be:

$$
\begin{aligned}
\sigma(x, t) & =\frac{\left[\mu_{p}(x)-\mu_{n}(x)\right]}{2} \frac{\partial D(x, t)}{\partial x} \\
+ & \sqrt{\left\{\frac{\left[\mu_{p}(x)+\mu_{n}(x)\right]}{2} \frac{\partial D(x, t)}{\partial x}\right\}^{2}+\sigma_{i n}(x)^{2}}
\end{aligned}
$$

which describes a nonlinear relation between the space-charge density and the overall local conductivity in the presence of a non-zero intrinsic conductivity.

\section{Limiting case of zero intrinsic conductivity}

Consider a case where $\sigma_{i n}(x) \rightarrow 0$ but $\sigma(x, t)$ remains finite. According to Eq. (15), either $\mu_{p}(x)$ or $\mu_{n}(x)$ has to be non-zero for $\sigma(x, t)$ to remain finite. From the definition of the intrinsic conductivity in Eq. (14), for $\sigma_{\text {in }}(x) \rightarrow 0$ we must have $C_{i n}(x) \rightarrow 0$, because the mobility sum $\left[\mu_{p}(x)+\mu_{n}(x)\right]$ is finite. For $C_{i n}(x) \rightarrow 0$, Eq. (20) becomes 


$$
\Delta p(x, t) \Delta n(x, t) \approx 0
$$

Multiplying both sides of Eq. (19) by $\Delta p(x, t)$ or $\Delta n(x, t)$ and then applying Eq. (35), we obtain

$$
\Delta p(x, t)\left[\Delta p(x, t)-\frac{1}{q} \frac{\partial D(x, t)}{\partial x}\right] \approx 0
$$

and

$$
\Delta n(x, t)\left[\Delta n(x, t)+\frac{1}{q} \frac{\partial D(x, t)}{\partial x}\right] \approx 0
$$

The solutions to Eqs. (36) and (37) are

$$
\Delta p(x, t) \approx 0, \Delta p(x, t) \approx \frac{1}{q} \frac{\partial D(x, t)}{\partial x}
$$

and

$$
\Delta n(x, t) \approx 0, \Delta n(x, t) \approx-\frac{1}{q} \frac{\partial D(x, t)}{\partial x}
$$

respectively. According to Eq. (19), however, only the following combinations of solutions are allowed.

$$
\Delta p(x, t) \approx 0 \text { and } \Delta n(x, t) \approx-\frac{1}{q} \frac{\partial D(x, t)}{\partial x}
$$

and

$$
\Delta n(x, t) \approx 0 \text { and } \Delta p(x, t) \approx \frac{1}{q} \frac{\partial D(x, t)}{\partial x}
$$

Since $C_{i n}(x) \rightarrow 0$, the quantities $\Delta p(x, t)$ and $\Delta n(x, t)$ effectively become the total free-carrier concentrations and must therefore be non-negative. Eqs. (40) and (41) thus lead to an important conclusion: At the limit of zero intrinsic conductivity, either p-type or n-type free charge-carriers become dominant, with the cases of

$$
\frac{\partial D(x, t)}{\partial x}>0
$$

and

$$
\frac{\partial D(x, t)}{\partial x}<0
$$

corresponding to the dominance of $p$-type and $n$-type free charge-carriers, respectively. This conclusion provides a linkage between two of the three independent assumptions, i.e. the absence of an intrinsic conductivity and the presence of only a single type of free charge- 
carriers, in the original derivation of the Mott-Gurney law: Although Eqs. (40) and (41) only conclude that the simultaneous presence of $p$-type and $n$-type free carriers is locally forbidden, this conclusion also holds globally across a dielectric sample for the case of a plane-parallel dielectric capacitor as described by the Mott-Gurney law. In the absence of diffusion currents, the total-current density $J$ is equal to the conduction-current density $J_{c}$, and they are both spatially continuous; For any non-zero total-current density $J$, the spacecharge density must be non-zero everywhere, otherwise there would be the case of $J=J_{c}=0$ somewhere in the sample. Globally, regions of positive space-charge density and regions of negative space-charge density cannot be present simultaneously in the dielectric sample, otherwise there would exist inter-regional boundaries of zero space-charge density at which $J=J_{c}=0$. It follows that the space-charge density must be of the same sign across the dielectric sample as well, which implies that the simultaneous presence of $p$-type and $n$-type free charge-carriers is also globally forbidden.

For $\sigma_{\text {in }}(x) \rightarrow 0$, Eq. (34) becomes

$$
\begin{aligned}
\sigma(x, t) & \approx \frac{\left[\mu_{p}(x)-\mu_{n}(x)\right]}{2} \frac{\partial D(x, t)}{\partial x} \\
+ & \frac{\left[\mu_{p}(x)+\mu_{n}(x)\right]}{2}\left|\frac{\partial D(x, t)}{\partial x}\right|
\end{aligned}
$$

If Eq. (42) holds, we have

$$
\begin{aligned}
\sigma(x, t) & \approx \frac{\left[\mu_{p}(x)-\mu_{n}(x)\right]}{2} \frac{\partial D(x, t)}{\partial x} \\
+ & \frac{\left[\mu_{p}(x)+\mu_{n}(x)\right]}{2} \frac{\partial D(x, t)}{\partial x} \\
& \approx \mu_{p}(x) \frac{\partial D(x, t)}{\partial x}
\end{aligned}
$$

Else if Eq. (43) holds, we have

$$
\begin{aligned}
\sigma(x, t) & \approx \frac{\left[\mu_{p}(x)-\mu_{n}(x)\right]}{2} \frac{\partial D(x, t)}{\partial x} \\
+ & \frac{\left[\mu_{p}(x)+\mu_{n}(x)\right]}{2}\left[-\frac{\partial D(x, t)}{\partial x}\right] \\
& \approx-\mu_{n}(x) \frac{\partial D(x, t)}{\partial x}
\end{aligned}
$$

Eqs. (45) and (46) together verify that, in the case of $\sigma_{i n}(x) \rightarrow 0$, the charge mobility in the expression for the generally time-dependent local conductivity $\sigma(x, t)$ is correctly equal to that of the dominant type of free carriers.

\section{Derivation of a general expression for the local diffusion-current density}

Apart from electrical conduction, i.e. the electric-field-driven flow of free charge-carriers, there also exists the possibility of charge diffusion due to the presence of a charge-density 
gradient or a temperature gradient, where the latter is often being referred to as the thermoelectric effect. For any bulk dielectric sample, consider the $i^{\text {th }}$ infinitesimal volume with dimensions $\Delta x, \Delta y$ and $\Delta z$. Along the $x$-direction, for example, the net force acting on the $p$-type free carriers within this infinitesimal volume is given by

$$
F_{p, i}=\left(P_{p, i-1}-P_{p, i+1}\right) \Delta y \Delta z
$$

where $P$ and the subscript $p$ denote the local pressure and $p$-type free charge-carriers, respectively. The total amount of charge carried by the $p$-type free carriers in this infinitesimal volume is

where

$$
Q_{p, i}=q C_{p} \Delta x \Delta y \Delta z
$$

$$
C_{p} \equiv C_{i n}+\Delta p
$$

is the total concentration of $p$-type free carriers for this infinitesimal volume. The corresponding force per unit charge, i.e. the mechanical counterpart of the electric field, is then given by

$$
\tilde{E}_{p, i} \equiv \frac{F_{p, i}}{Q_{p, i}}=-\frac{\left(P_{p, i+1}-P_{p, i-1}\right)}{q C_{p} \Delta x}
$$

And for the case of conduction by $n$-type free carriers, we have

$$
\tilde{E}_{n, i} \equiv \frac{F_{n, i}}{Q_{n, i}}=-\frac{\left(P_{n, i+1}-P_{n, i-1}\right)}{\left(-q C_{n}\right) \Delta x}
$$

where

$$
C_{n} \equiv C_{i n}+\Delta n
$$

is the total concentration of $n$-type free carriers. In the continuum limit, Eqs. (50) and (51) can be replaced by

$$
\tilde{E}_{p}(x, t)=-\frac{1}{q C_{p}(x, t)} \frac{\partial P_{p}(x, t)}{\partial x}
$$

and

$$
\tilde{E}_{n}(x, t)=-\frac{1}{\left[-q C_{n}(x, t)\right]} \frac{\partial P_{n}(x, t)}{\partial x}
$$

respectively. The diffusion-current density is then given by

$$
\begin{aligned}
J_{d}(x, t)=q[ & \mu_{p}(x) C_{p}(x, t) \tilde{E}_{p}(x, t) \\
& \left.+\mu_{n}(x) C_{n}(x, t) \tilde{E}_{n}(x, t)\right] \\
=- & {\left[\mu_{p}(x) \frac{\partial P_{p}(x, t)}{\partial x}+\left[-\mu_{n}(x)\right] \frac{\partial P_{n}(x, t)}{\partial x}\right] }
\end{aligned}
$$


Using an ideal-gas approximation for each of the two types of free charge-carriers, i.e.

$$
P_{p}(x, t)=k_{B} T(x, t) C_{p}(x, t)
$$

and

$$
P_{n}(x, t)=k_{B} T(x, t) C_{n}(x, t)
$$

where $k_{B}$ is the Boltzmann constant and $T(x, t)$ is a generally position- and time-dependent local temperature, the diffusion-current density can be expressed as

$$
\begin{aligned}
J_{d}(x, t)= & -k_{B} T(x, t)\left[\begin{array}{l}
\mu_{p}(x) \frac{\partial C_{p}(x, t)}{\partial x} \\
+\left[-\mu_{n}(x)\right] \frac{\partial C_{n}(x, t)}{\partial x}
\end{array}\right] \\
& -k_{B} \frac{\partial T(x, t)}{\partial x}\left[\mu_{p}(x) C_{p}(x, t)+\left[-\mu_{n}(x)\right] C_{n}(x, t)\right]
\end{aligned}
$$

where

$$
D_{o, p}(x, t) \equiv \frac{\mu_{p}(x) k_{B} T(x, t)}{q}
$$

and

$$
D_{o, n}(x, t) \equiv \frac{\left[-\mu_{n}(x)\right] k_{B} T(x, t)}{(-q)}
$$

are the Einstein relations for the local diffusion coefficients of $p$-type and $n$-type free charge-carriers, respectively. Using the definitions in Eqs. (49) and (52), Eq. (58) can be rewritten as

$$
\begin{array}{r}
J_{d}(x, t)=-k_{B} T(x, t)\left[\begin{array}{l}
{\left[\mu_{p}(x)-\mu_{n}(x)\right] \frac{d C_{i n}(x)}{d x}} \\
+\mu_{p}(x) \frac{\partial \Delta p(x, t)}{\partial x}-\mu_{n}(x) \frac{\partial \Delta n(x, t)}{\partial x}
\end{array}\right] \\
-k_{B} \frac{\partial T(x, t)}{\partial x}\left[\begin{array}{l}
{\left[\mu_{p}(x)-\mu_{n}(x)\right] C_{i n}(x)} \\
+\mu_{p}(x) \Delta p(x, t)-\mu_{n}(x) \Delta n(x, t)
\end{array}\right]
\end{array}
$$

From Eqs. (21) and (22), we obtain

$$
\begin{aligned}
\Delta p(x, t) & =\frac{1}{2 q} \frac{\partial D(x, t)}{\partial x}-C_{i n}(x) \\
& +\sqrt{\left[\frac{1}{2 q} \frac{\partial D(x, t)}{\partial x}\right]^{2}+C_{i n}(x)^{2}}
\end{aligned}
$$


and

$$
\begin{aligned}
\Delta n(x, t) & =-\frac{1}{2 q} \frac{\partial D(x, t)}{\partial x}-C_{i n}(x) \\
& +\sqrt{\left[\frac{1}{2 q} \frac{\partial D(x, t)}{\partial x}\right]^{2}+C_{i n}(x)^{2}}
\end{aligned}
$$

Differentiating Eqs. (62) and (63) with respect to $x$ yields

$$
\begin{aligned}
& \frac{\partial \Delta p(x, t)}{\partial x}=\frac{1}{2 q} \frac{\partial^{2} D(x, t)}{\partial x^{2}}-\frac{d C_{i n}(x)}{d x} \\
& +\left\{\sqrt{\left[\frac{1}{2 q} \frac{\partial D(x, t)}{\partial x}\right]^{2}+C_{i n}(x)^{2}}\right\}^{-1} \\
& \times\left[\frac{1}{4 q^{2}} \frac{\partial D(x, t)}{\partial x} \frac{\partial^{2} D(x, t)}{\partial x^{2}}+C_{i n}(x) \frac{d C_{i n}(x)}{d x}\right]
\end{aligned}
$$

and

$$
\begin{aligned}
& \frac{\partial \Delta n(x, t)}{\partial x}=-\frac{1}{2 q} \frac{\partial^{2} D(x, t)}{\partial x^{2}}-\frac{d C_{i n}(x)}{d x} \\
& +\left\{\sqrt{\left[\frac{1}{2 q} \frac{\partial D(x, t)}{\partial x}\right]^{2}+C_{i n}(x)^{2}}\right\}^{-1} \\
& \times\left[\frac{1}{4 q^{2}} \frac{\partial D(x, t)}{\partial x} \frac{\partial^{2} D(x, t)}{\partial x^{2}}+C_{i n}(x) \frac{d C_{i n}(x)}{d x}\right]
\end{aligned}
$$

respectively. Putting Eqs. (62) to (65) into Eq. (61), we obtain a general expression for the local diffusion-current density:

$$
J_{d}(x, t)=J_{d 1}(x, t)+J_{d 2}(x, t)
$$

where

$$
\begin{gathered}
J_{d 1}(x, t) \equiv-\frac{k_{B} T(x, t)\left[\mu_{p}(x)+\mu_{n}(x)\right]}{2 q} \frac{\partial^{2} D(x, t)}{\partial x^{2}} \\
-\left[\mu_{p}(x)-\mu_{n}(x)\right] k_{B} T(x, t) \times\left\{\sqrt{\left[\frac{1}{2 q} \frac{\partial D(x, t)}{\partial x}\right]^{2}+C_{i n}(x)^{2}}\right\}^{-1} \\
\times\left[\frac{1}{4 q^{2}} \frac{\partial D(x, t)}{\partial x} \frac{\partial^{2} D(x, t)}{\partial x^{2}}+C_{i n}(x) \frac{d C_{i n}(x)}{d x}\right]
\end{gathered}
$$


and

$$
J_{d 2}(x, t) \equiv-k_{B} \frac{\partial T(x, t)}{\partial x}\left[\begin{array}{l}
\frac{\left[\mu_{p}(x)+\mu_{n}(x)\right]}{2 q} \frac{\partial D(x, t)}{\partial x} \\
+\left[\mu_{p}(x)-\mu_{n}(x)\right] \\
\times \sqrt{\left[\frac{1}{2 q} \frac{\partial D(x, t)}{\partial x}\right]^{2}+C_{i n}(x)^{2}}
\end{array}\right]
$$

Eq. (67) denotes a contribution to the diffusion current from the presence of a gradient in the space-charge density or in the intrinsic free-carrier concentration, while Eq. (68) denotes a contribution from the presence of a temperature gradient. At the limit of zero intrinsic conductivity, we have $C_{i n}(x) \rightarrow 0$ as explained in the beginning of the previous section. Eqs. (67) and (68) are then reduced to

$$
\begin{aligned}
J_{d 1}(x, t) \approx- & \frac{\left[\mu_{p}(x)+\mu_{n}(x)\right] k_{B} T(x, t)}{2 q} \frac{\partial^{2} D(x, t)}{\partial x^{2}} \\
& -\frac{\left[\mu_{p}(x)-\mu_{n}(x)\right] k_{B} T(x, t)}{2 q} \frac{\frac{\partial D(x, t)}{\partial x} \frac{\partial^{2} D(x, t)}{\partial x^{2}}}{\left|\frac{\partial D(x, t)}{\partial x}\right|}
\end{aligned}
$$

and

$$
J_{d 2}(x, t) \approx-k_{B} \frac{\partial T(x, t)}{\partial x}\left[\begin{array}{l}
\frac{\left[\mu_{p}(x)+\mu_{n}(x)\right]}{2 q} \frac{\partial D(x, t)}{\partial x} \\
+\frac{\left[\mu_{p}(x)-\mu_{n}(x)\right]}{2 q} \mid \frac{\partial D(x, t)}{\partial x}
\end{array}\right]
$$

respectively. Similar to the case of Eq. (44), for Eqs. (69) and (70) we can also verify that in the case of $\sigma_{i n}(x) \rightarrow 0$ the charge mobility is correctly equal to that of the dominant type of free carriers. Following Eqs. (69) and (70), if Eq. (42) is satisfied, we have

$$
\begin{aligned}
J_{d 1}(x, t) \approx & -\frac{\left[\mu_{p}(x)+\mu_{n}(x)\right] k_{B} T(x, t)}{2 q} \frac{\partial^{2} D(x, t)}{\partial x^{2}} \\
& -\frac{\left[\mu_{p}(x)-\mu_{n}(x)\right] k_{B} T(x, t)}{2 q} \frac{\frac{\partial D(x, t)}{\partial x} \frac{\partial^{2} D(x, t)}{\partial x^{2}}}{\left[+\frac{\partial D(x, t)}{\partial x}\right]} \\
\approx & -\frac{\mu_{p}(x) k_{B} T(x, t)}{q} \frac{\partial^{2} D(x, t)}{\partial x^{2}}
\end{aligned}
$$

and 


$$
\begin{aligned}
J_{d 2}(x, t) & \approx-k_{B} \frac{\partial T(x, t)}{\partial x}\left[\begin{array}{l}
\frac{\left[\mu_{p}(x)+\mu_{n}(x)\right]}{2 q} \frac{\partial D(x, t)}{\partial x} \\
+\frac{\left[\mu_{p}(x)-\mu_{n}(x)\right]}{2 q}\left[+\frac{\partial D(x, t)}{\partial x}\right]
\end{array}\right] \\
& \approx-\frac{\mu_{p}(x) k_{B}}{q} \frac{\partial D(x, t)}{\partial x} \frac{\partial T(x, t)}{\partial x}
\end{aligned}
$$

Else if Eq. (43) is satisfied, we have

$$
\begin{aligned}
J_{d 1}(x, t) \approx- & \frac{\left[\mu_{p}(x)+\mu_{n}(x)\right] k_{B} T(x, t)}{2 q} \frac{\partial^{2} D(x, t)}{\partial x^{2}} \\
& -\frac{\left[\mu_{p}(x)-\mu_{n}(x)\right] k_{B} T(x, t)}{2 q} \frac{\frac{\partial D(x, t)}{\partial x} \frac{\partial^{2} D(x, t)}{\partial x^{2}}}{\left[-\frac{\partial D(x, t)}{\partial x}\right]} \\
\approx & -\frac{\mu_{n}(x) k_{B} T(x, t)}{q} \frac{\partial^{2} D(x, t)}{\partial x^{2}}
\end{aligned}
$$

and

$$
\begin{aligned}
J_{d 2}(x, t) & \approx-k_{B} \frac{\partial T(x, t)}{\partial x}\left[\begin{array}{l}
\frac{\left[\mu_{p}(x)+\mu_{n}(x)\right]}{2 q} \frac{\partial D(x, t)}{\partial x} \\
+\frac{\left[\mu_{p}(x)-\mu_{n}(x)\right]}{2 q}\left[-\frac{\partial D(x, t)}{\partial x}\right]
\end{array}\right] \\
& \approx-\frac{\mu_{n}(x) k_{B}}{q} \frac{\partial D(x, t)}{\partial x} \frac{\partial T(x, t)}{\partial x}
\end{aligned}
$$

\section{Alternative derivation of the general local conductivity expression}

We begin our alternative derivation of the general local conductivity expression in Eq. (34) by identifying the following quantities that appear in the conductivity expression:

$$
\mu^{\prime}(x) \equiv \frac{\mu_{p}(x)-\mu_{n}(x)}{2}
$$

and

$$
\mu^{\prime \prime}(x) \equiv \frac{\mu_{p}(x)+\mu_{n}(x)}{2}
$$

The drift velocities of $p$-type and $n$-type free carriers can then be expressed as

$$
v_{p}(x, t)=\mu_{p}(x) E(x, t)=\mu^{\prime}(x) E(x, t)+\mu^{\prime \prime}(x) E(x, t)
$$

and 


$$
v_{n}(x, t)=-\mu_{n}(x) E(x, t)=\mu^{\prime}(x) E(x, t)-\mu^{\prime \prime}(x) E(x, t)
$$

where

$$
\mu^{\prime \prime}(x) \geq\left|\mu^{\prime}(x)\right| \geq 0
$$

and $\mu^{\prime}(x)$ can be positive or negative. In this description, both $p$-type and $n$-type free carriers share the same velocity component $\mu^{\prime}(x) E(x, t)$, with the presence of the additional velocity components $\mu^{\prime \prime}(x) E(x, t)$ and $-\mu^{\prime \prime}(x) E(x, t)$ for $p$-type and $n$-type free carriers, respectively. The generally time-dependent local electrical conductivity can then be expressed as a sum of contributions from the velocity components $\mu^{\prime}(x) E(x, t)$ and $\pm \mu^{\prime \prime}(x) E(x, t)$ :

$$
\begin{aligned}
\sigma(x, t)=q \mu^{\prime}(x) & {\left[C_{p}(x, t)-C_{n}(x, t)\right] } \\
& +q \mu^{\prime \prime}(x)\left[C_{p}(x, t)+C_{n}(x, t)\right]
\end{aligned}
$$

According to Gauss' law, the density of free space-charge is given by

$$
\rho_{q}(x, t)=\frac{\partial D(x, t)}{\partial x}=q\left[C_{p}(x, t)-C_{n}(x, t)\right]
$$

In the absence of free space-charge, i.e. $\rho_{q}(x, t)=0$, both $C_{p}(x, t)$ and $C_{n}(x, t)$ are by definition equal to the intrinsic free-carrier concentration $C_{i n}(x)$, and the electrical conductivity $\sigma(x, t)$ would then be equal to the intrinsic conductivity

$$
\sigma_{\text {in }}(x) \equiv 2 q \mu^{\prime \prime}(x) C_{i n}(x)
$$

according to Eq. (80).

Consider the reversible generation and recombination of $p$-type and $n$-type free carriers:

$$
1 \text { source particle } \leftarrow \rightarrow 1 p \text {-type free carrier }+1 n \text {-type free carrier }
$$

As described right below Eq. (18), the rate of free-carrier generation is assumed to be equal to the rate of free-carrier recombination due to a "heat balance" condition, and the rate of each of these processes is assumed to be proportional to the product of the "reactants". Following these, for $C_{s}(x, t)$ being the concentration of the source particles for free-carrier generation (e.g. valence electrons or molecules) we have

$$
K_{g} C_{s}(x, t)=K_{r} C_{p}(x, t) C_{n}(x, t)
$$

where $K_{g}$ and $K_{r}$ are, respectively, the rate constants for the generation and recombination of free carriers. If the conditions

$$
C_{p}(x, t)<<C_{s}(x, t)
$$

and

$$
C_{n}(x, t)<<C_{s}(x, t)
$$

hold for a dielectric insulator such that 


$$
C_{s}(x, t) \approx C_{s}(x)
$$

i.e. the concentration of source particles for free-carrier generation has an insignificant fluctuation with time and is practically a material-pertaining property, we have

$$
K_{g} C_{s}(x) \approx K_{r} C_{p}(x, t) C_{n}(x, t) \approx K_{r} C_{i n}(x)^{2}
$$

which implies

$$
C_{p}(x, t) C_{n}(x, t) \approx C_{i n}(x)^{2}
$$

As an example, we show that this mass-action approximation is valid for a dielectric insulator which has holes and free electrons as its $p$-type and $n$-type free charge-carriers, respectively, and which has valence electrons as its source particles: A hole is by definition equivalent to a missing valence electron. At anywhere inside the dielectric sample, the generation and annihilation of a hole correspond, by definition, to the annihilation and generation of a valence electron, respectively, and the flow-in and flow-out of a hole are, respectively, by definition equivalent to the flow-out and flow-in of a valence electron in the opposite directions. Therefore,

$$
\frac{\partial C_{p}(x, t)}{\partial t}=-\frac{\partial C_{s}(x, t)}{\partial t}
$$

so that the total concentration of holes and valence electrons is given by

$$
C_{p+s}(x) \equiv C_{p}(x, t)+C_{s}(x, t)
$$

Eq. (83) can then be written as

$$
K_{g}\left[C_{p+s}(x)-C_{p}(x, t)\right]=K_{r} C_{p}(x, t) C_{n}(x, t)
$$

For the case of zero space charge where $C_{p}(x, t)=C_{n}(x, t)=C_{i n}(x)$, we have

$$
K_{g}\left[C_{p+s}(x)-C_{i n}(x)\right]=K_{r} C_{i n}(x)^{2}
$$

Define a material paramter

$$
\alpha(x) \equiv \frac{C_{\text {in }}(x)}{C_{p+s}(x)} \leq 1
$$

and consider the limit of $\alpha(x) \rightarrow 0$ for the case of a dielectric insulator. Combining Eqs. (91) to (93), we obtain the mass-action relation in Eq. (88):

$$
\begin{aligned}
& C_{p}(x, t) C_{n}(x, t) \\
& =\operatorname{limit}_{\alpha(x) \rightarrow 0} \frac{C_{i n}(x)\left[C_{i n}(x)-\alpha(x) C_{p}(x, t)\right]}{[1-\alpha(x)]}=C_{i n}(x)^{2}
\end{aligned}
$$

Going back to our derivation of the conductivity expression, we notice that Eqs. (81) and (88) together imply 


$$
C_{p}(x, t)^{2}-\frac{\rho_{q}(x, t)}{q} C_{p}(x, t)-C_{i n}(x)^{2} \approx 0
$$

and

$$
C_{n}(x, t)^{2}+\frac{\rho_{q}(x, t)}{q} C_{n}(x, t)-C_{i n}(x)^{2} \approx 0
$$

from which we obtain

$$
\begin{aligned}
& C_{p}(x, t) \approx \frac{1}{2}\left[\frac{\rho_{q}(x, t)}{q}+\sqrt{\left[\frac{\rho_{q}(x, t)}{q}\right]^{2}+4 C_{i n}(x)^{2}}\right] \geq 0 \\
& C_{n}(x, t) \approx \frac{1}{2}\left[-\frac{\rho_{q}(x, t)}{q}+\sqrt{\left[\frac{\rho_{q}(x, t)}{q}\right]^{2}+4 C_{i n}(x)^{2}}\right] \geq 0
\end{aligned}
$$

and

$$
C_{p}(x, t)+C_{n}(x, t) \approx \sqrt{\left[\frac{\rho_{q}(x, t)}{q}\right]^{2}+4 C_{i n}(x)^{2}}
$$

Using Eqs. (80) to (82) as well as Eq. (99), we obtain the following expression for the generally time-dependent local electrical conductivity:

$$
\sigma(x, t) \approx \mu^{\prime}(x) \rho_{q}(x, t)+\sqrt{\left[\mu^{\prime \prime}(x) \rho_{q}(x, t)\right]^{2}+\sigma_{i n}(x)^{2}}
$$

By defining the reduced paramters

$$
\begin{gathered}
\sigma^{*}(x, t) \equiv \frac{\sigma(x, t)}{\sigma_{\text {in }}(x)} \\
\mu^{*}(x) \equiv \frac{\mu^{\prime}(x)}{\mu^{\prime \prime}(x)} \in\{-1,+1\}
\end{gathered}
$$

and

$$
\rho_{q}^{*}(x, t) \equiv \frac{\rho_{q}(x, t)}{2 q C_{i n}(x)}
$$

Eq. (100) can be expressed in a simpler form:

$$
\sigma^{*}(x, t) \approx \mu^{*}(x) \rho_{q}^{*}(x, t)+\sqrt{\left[\rho_{q}^{*}(x, t)\right]^{2}+1}
$$

For the limiting case of zero intrinsic conductivity with $C_{i n}(x) \rightarrow 0$, Eqs. (97) and (98) can be rewritten as 


$$
C_{p}(x, t) \approx \frac{1}{2}\left[\frac{\rho_{q}(x, t)}{q}+\left|\frac{\rho_{q}(x, t)}{q}\right|\right]
$$

and

$$
C_{n}(x, t) \approx \frac{1}{2}\left[-\frac{\rho_{q}(x, t)}{q}+\left|\frac{\rho_{q}(x, t)}{q}\right|\right]
$$

respectively, which imply the dominance of either type of free carriers: If $\rho_{q}(x, t)>0$, we have

$$
C_{p}(x, t) \approx \frac{\rho_{q}(x, t)}{q} \text { and } C_{n}(x, t) \approx 0
$$

Else if $\rho_{q}(x, t)<0$, we have

$$
C_{p}(x, t) \approx 0 \text { and } C_{n}(x, t) \approx-\frac{\rho_{q}(x, t)}{q}
$$

\section{Conclusions and future work}

In this Chapter, a generalized theory for space-charge-limited conduction (SCLC) in ferroelectrics and other solid dielectrics, which we have originally developed to account for the peculiar observation of polarization offsets in compositionally graded ferroelectric films, is presented in full. The theory is a generalization of the conventional steady-state trap-free SCLC model, as described by the Mott-Gurney law, to include (i) the presence of two opposite types of free carriers: $p$-type and $n$-type, (ii) the presence of a finite intrinsic (Ohmic) conductivity, (iii) any possible field- and time-dependence of the dielectric permittivity, and (iv) any possible time dependence of the dielectric system under study. Expressions for the local conductivity as well as for the local diffusion-current density were derived through a mass-action approximation for which a detailed theoretical justification is provided in this Chapter. It was found that, in the presence of a finite intrinsic conductivity, both the local conductivity and the local diffusion-current density are related to the spacecharge density in a nonlinear fashion, as described by Eqs. (34), (66), (67) and (68), where the local diffusion-current density is generally described as a sum of contributions from the presence of a charge-density gradient and of a temperature gradient. At the limit of zero intrinsic conductivity, it was found that either $p$-type or $n$-type free carriers are dominant. This conclusion provides a linkage between the independent assumptions of (i) a single carrier type and (ii) a negligible intrinsic conductivity in the conventional steady-state SCLC model. For any given space-charge density, it was also verified that the expressions we have derived correctly predict the dominant type of free carriers at the limit of zero intrinsic conductivity.

Future work should be carried out along at least three possible directions: (i) As a further application of this general local conductivity expression, further numerical investigations should be carried out on how charge actually flows inside a compositionally graded ferroelectric film. This would provide answers to interesting questions like: Does a graded ferroelectric system exhibit any kind of charge-density waves upon excitation by an 
alternating electric field? What are the physical factors (dielectric permittivity, carrier mobility, etc.) that could limit or enhance the degree of asymmetry in the SCLC currents of a graded ferroelectric film? The latter question has been partially answered by ourselves (Zhou et al., 2005b), where we have theoretically found that the observation of polarization offsets, i.e. the onset of asymmetric SCLC, in a compositionally graded ferroelectric film is conditional upon the presence of relatively large gradients in the polarization and in the dielectric permittivity. Certainly, a detailed understanding of the mechanism of asymmetric electrical conduction in such a graded ferroelectric film would also provide insights into the designing of new types of electrical diodes or rectifiers. The recently derived expression for the local diffusion-current density, as first presented in this Book Chapter (Eqs. (66) to (68)), has also opened up a new dimension for further theoretical investigations: Using this expression, the effect of charge diffusion in the presence of a charge-density gradient or a temperature gradient can be taken into account as well, and a whole new range of problems can be studied. For example, it would be interesting to know whether asymmetric electrical conduction would also occur if a compositionally graded ferroelectric film is driven by a sinusoidal applied temperature difference instead of a sinusoidal applied voltage. In this case, one also needs to take into account the temperature dependence of the various system parameters like the remanent polarization and the dielectric permittivity. The theoretical predictions should then be compared against any available experimental results. (ii) Going back to the generalized SCLC theory itself, it would be important to look for possible experimental verifications of the general local conductivity expression, and to establish a set of physical conditions under which the conductivity expression and the corresponding mass-action approximation are valid. Theoretical predictions from the conductivity expression should be made for real experimental systems and then be compared with available experimental results. It would also be worthwhile to generalize the mass-action approximation, and hence the corresponding local conductivity expression, to other cases where the charge of the free carriers, or the stoichiometric ratio between the concentrations of $p$-type and $n$-type free carriers in the generationrecombination processes, is different. (iii) In the derivation of the Mott-Gurney law $J \sim V^{2}$, the boundary conditions $E_{p}(0)=0$ and $E_{n}(L)=0$ were employed to describe the cases of conduction by $p$-type and $n$-type free carriers, respectively. If we keep $E_{p}(0)$ or $E_{n}(L)$ as a variable throughout the derivation, an expression of $J$ as a function of $E_{p}(0)$ or $E_{n}(L)$ can be obtained and it can be shown that both the boundary conditions $E_{p}(0)=0$ and $E_{n}(L)=0$ correspond to a state of maximum current density. As an example, for the case of conduction by $p$-type free carriers, we have (Fig. 2)

$$
\begin{aligned}
j_{p} \equiv \frac{J_{p} L^{3}}{\mu_{p} \varepsilon V^{2}} & =\left[\frac{9-12 e_{p}(0)^{2}}{16}\right] \\
& +\sqrt{\left[\frac{9-12 e_{p}(0)^{2}}{16}\right]^{2}+\frac{3}{4} e_{p}(0)^{3}\left[1-e_{p}(0)\right]}
\end{aligned}
$$

where $e_{p}(0) \equiv E_{p}(0) L / V$. If we consider our general local conductivity expression which takes into account the presence of a finite intrinsic conductivity and the simultaneous presence of 
$p$-type and $n$-type free carriers, it would be important to know whether this maximumcurrent principle can be generally applied to obtain the system's boundary conditions.

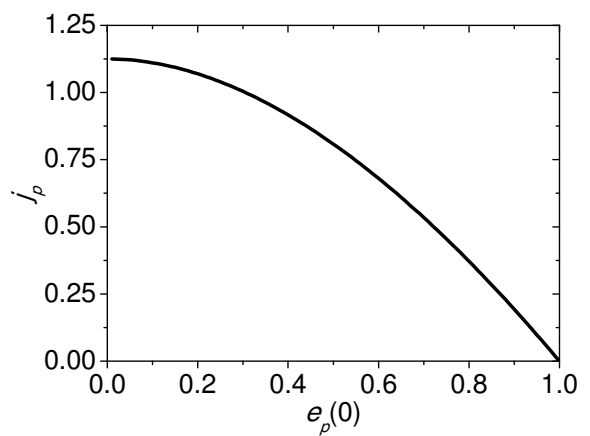

Fig. 2. Plot of $j_{p}$ against $e_{p}(0)$, showing a maximum of $j_{p}$ at $e_{p}(0)=0$.

\section{Acknowledgments}

Stimulating discussions with Prof. Franklin G. Shin, Dr. Chi-Hang Lam and Dr. Yan Zhou are gratefully acknowledged.

\section{References}

Alpay, S. P.; Ban, Z. G. \& Mantese, J. V. (2003). Thermodynamic Analysis of Temperaturegraded Ferroelectrics. Applied Physics Letters, Vol. 82, pp. 1269 - 1271 (February 2003), ISSN 1077-3118

Bao, D.; Mizutani, N.; Zhang, L. \& Yao, X. (2001). Composition Gradient Optimization and Electrical Characterization of $(\mathrm{Pb}, \mathrm{Ca}) \mathrm{TiO}_{3}$ Thin Films. Journal of Applied Physics, Vol. 89, pp. 801 - 803 (January 2001), ISSN 1089-7550

Bao, D.; Wakiya, N.; Shinozaki, K.; Mizutani, N. \& Yao, X. (2001). Abnormal Ferroelectric Properties of Compositionally Graded $\mathrm{Pb}(\mathrm{Zr}, \mathrm{Ti}) \mathrm{O}_{3}$ Thin Films with $\mathrm{LaNiO}_{3}$ Bottom Electrodes. Journal of Applied Physics, Vol. 90, pp. 506 - 508 (July 2001), ISSN 10897550

Bao, D.; Mizutani, N.; Yao, X. \& Zhang, L. (2000). Structural, Dielectric, and Ferroelectric Properties of Compositionally Graded $(\mathrm{Pb}, \mathrm{La}) \mathrm{TiO}_{3}$ Thin Films with Conductive $\mathrm{LaNiO}_{3}$ Bottom Electrodes. Applied Physics Letters, Vol. 77, pp. 1041 - 1043 (August 2000), ISSN 1077-3118

Bao, D.; Mizutani, N.; Yao, X. \& Zhang, L. (2000). Dielectric and Ferroelectric Properties of Compositionally Graded (Pb,La) $\mathrm{TiO}_{3}$ Thin Films on $\mathrm{Pt} / \mathrm{Ti} / \mathrm{SiO}_{2} / \mathrm{Si}$ Substrates. Applied Physics Letters, Vol. 77, pp. 1203 - 1205 (August 2000), ISSN 1077-3118

Bao, D.; Yao, X. \& Zhang, L. (2000). Dielectric Enhancement and Ferroelectric Anomaly of Compositionally Graded $(\mathrm{Pb}, \mathrm{Ca}) \mathrm{TiO}_{3}$ Thin Films Derived by a Modified Sol-gel Technique. Applied Physics Letters, Vol. 76, pp. 2779 - 2781 (May 2000), ISSN 10773118 
Bouregba, R.; Poullain, G.; Vilquin, B. \& Le Rhun, G. (2003). Asymmetrical Leakage Currents as a Possible Origin of the Polarization Offsets Observed in Compositionally Graded Ferroelectric Films. Journal of Applied Physics, Vol. 93, pp. 5583 - 5591 (May 2003), ISSN 1089-7550

Brazier, M.; McElfresh, M. \& Mansour, S. (1998). Unconventional Hysteresis Behavior in Compositionally Graded $\mathrm{Pb}(\mathrm{Zr}, \mathrm{Ti}) \mathrm{O}_{3}$ Thin Films. Applied Physics Letters, Vol. 72, pp. 1121 - 1123 (March 1998) , ISSN 1077-3118

Brazier, M.; McElfresh, M. \& Mansour, S. (1999). Origin of Anomalous Polarization Offsets in Compositionally Graded $\mathrm{Pb}(\mathrm{Zr}, \mathrm{Ti}) \mathrm{O}_{3}$ Thin Films. Applied Physics Letters, Vol. 74, pp. 299 - 301 (January 1999), ISSN 1077-3118

Carbone, A.; Kotowska, B. K. \& Kotowski, D. (2005). Space-Charge-Limited Current Fluctuations in Organic Semiconductors. Physical Review Letters, Vol. 95, No. 236601 (December 2005), ISSN 1079-7114

Chan, H. K.; Lam, C. H. \& Shin, F. G. (2004). Time-dependent Space-charge-limited Conduction as a Possible Origin of the Polarization Offsets Observed in Compositionally Graded Ferroelectric Films. Journal of Applied Physics, Vol. 95, pp. 2665 - 2671 (March 2004), ISSN 1089-7550 [The currently valid email address of the corresponding author Ho-Kei Chan is "epkeiyeah@yahoo.com.hk"]

Chan, H. K.; Zhou, Y.; Lam, C. H. \& Shin, F. G. (2007). Theory of Time-dependent Spacecharge-limited Conduction, Proceedings of IEEE $20079^{\text {th }}$ International Conference on Solid Dielectrics, pp. 35 - 38, ISBN 1-4244-0751-6, Winchester, United Kingdom, July 8-13, 2007 [Typo in the paper: Eq. (5) should be referred to as the MottGurney law]

Chen, Z.; Arita, K.; Lim, M. \& Paz De Araujo, C. A. (1999). Graded PZT Thin Film Capacitors with Stoichimetric Variation by MOD Technique. Integrated Ferroelectrics, Vol. 24, pp. 181 - 188 (August 1999), ISSN 1607-8489

Coelho, R. (1979). Physics of Dielectrics for the Engineer, Elsevier Scientific, ISBN 0444417559, New York, United States of America

Fellberg, W.; Mantese, J. V.; Schubring, N. W. \& Micheli, A. L. (2001). Origin of the "up", "down" Hysteresis Offsets Observed from Polarization-graded Ferroelectric Materials. Applied Physics Letters, Vol. 78, pp. 524 - 526 (January 2001), ISSN 10773118

Laha, A. \& Krupanidhi, S. B. (2002). Leakage Current Conduction of Pulsed Excimer Laser Ablated $\mathrm{BaBi}_{2} \mathrm{Nb}_{2} \mathrm{O}_{9}$ Thin Films. Journal of Applied Physics, Vol. 92, pp. $415-420$ (July 2002), ISSN 1089-7550

Mantese, J. V. \& Alpay, S. P. (2005). Graded Ferroelectrics, Transpacitors and Transponents, Springer, ISBN 0387233113, New York, United States of America

Mantese, J. V. ; Schubring, N. W.; Micheli, A. L.; Catalan, A. B.; Mohammed, M. S.; Naik, R. \& Auner, G. W. (1997). Slater Model Applied to Polarization Graded Ferroelectrics. Applied Physics Letters, Vol. 71, pp. 2047 - 2049 (October 1997), ISSN 1077-3118

Matsuzaki, T. \& Funakubo, H. (1999). Preparation and Characterization of $\mathrm{Pb}(\mathrm{Nb}, \mathrm{Ti}) \mathrm{O}_{3}$ Thin Films by Metalorganic Chemical Vapor Deposition. Journal of Applied Physics, Vol. 86, pp. 4559 - 4564 (October 1999), ISSN 1089-7550

Okatan, M. B.; Mantese, J. V. \& Alpay, S. P. (2010). Effect of Space Charge on the Polarization Hysteresis Characteristics of Monolithic and Compositionally Graded Ferroelectrics Acta Materialia, Vol. 58, pp. 39 - 48 (January 2010), ISSN 1359-6454 
Pope, M. \& Swemberg, C. E. (1998). Electronic Processes in Organic Crystals and Polymers, Oxford University Press, ISBN 0195129636, Oxford, United Kingdom

Poullain, G.; Bouregba, R.; Vilquin, B.; Le Rhun, G. \& Murray, H. (2002). Graded Ferroelectric Thin Films: Possible Origin of the Shift Along the Polarization Axis. Applied Physics Letters, Vol. 81, pp. 5015 - 5017 (December 2002), ISSN 10773118

Sawyer, C. B. \& Tower, C. H. (1930). Rochelle Salt as a Dielectric. Physical Review, Vol. 35, pp. 269 - 273 (February 1930), ISSN 0031-899X

Schubring, N. W.; Mantese, J. V.; Micheli, A. L.; Catalan, A. B. \& Lopez, R. J. (1992). Charge Pumping and Pseudo-pyroelectric Effect in Active Ferroelectric Relaxor-type Films. Physical Review Letters, Vol. 68, pp. 1778 - 1781 (March 1992), ISSN 1079-7114

Suh, K. S.; Kim, J. E.; Oh, W. J.; Yoon, H. G. \& Takada, T. (2000). Charge Distribution and Conduction Characteristics of 2-vinylpyridine-grafted Polyethylenes. Journal of Applied Physics, Vol. 87, pp. 7333 - 7337 (May 2000), ISSN 1089-7550

Zhou, Y.; Chan, H. K.; Lam, C. H. \& Shin, F. G. (2005). Mechanisms of Imprint Effect on Ferroelectric Thin Films. Journal of Applied Physics, Vol. 98, No. 024111 (July 2005), ISSN 1089-7550

Zhou, Y.; Chan, H. K.; Lam, C. H. \& Shin, F. G. (2005). Effects of Polarization and Permittivity Gradients and Other Parameters on the Anomalous Vertical Shift Behavior of Graded Ferroelectric Thin Films. Journal of Applied Physics, Vol. 98, No. 034105 (August 2005), ISSN 1089-7550 


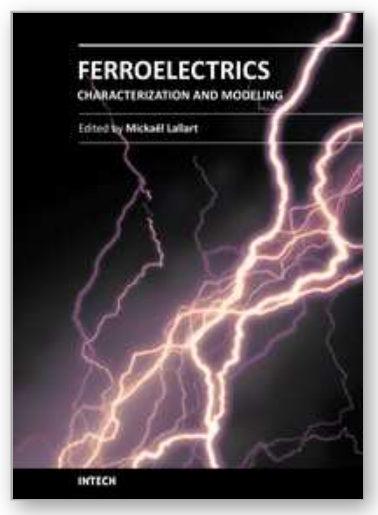

\author{
Ferroelectrics - Characterization and Modeling \\ Edited by Dr. MickaÃ«l Lallart
}

ISBN 978-953-307-455-9

Hard cover, 586 pages

Publisher InTech

Published online 23, August, 2011

Published in print edition August, 2011

Ferroelectric materials have been and still are widely used in many applications, that have moved from sonar towards breakthrough technologies such as memories or optical devices. This book is a part of a four volume collection (covering material aspects, physical effects, characterization and modeling, and applications) and focuses on the characterization of ferroelectric materials, including structural, electrical and multiphysic aspects, as well as innovative techniques for modeling and predicting the performance of these devices using phenomenological approaches and nonlinear methods. Hence, the aim of this book is to provide an up-to-date review of recent scientific findings and recent advances in the field of ferroelectric system characterization and modeling, allowing a deep understanding of ferroelectricity.

\title{
How to reference
}

In order to correctly reference this scholarly work, feel free to copy and paste the following:

Ho-Kei Chan (2011). A General Conductivity Expression for Space-Charge-Limited Conduction in Ferroelectrics and Other Solid Dielectrics, Ferroelectrics - Characterization and Modeling, Dr. Micka Ã«I Lallart (Ed.), ISBN: 978-953-307-455-9, InTech, Available from: http://www.intechopen.com/books/ferroelectricscharacterization-and-modeling/a-general-conductivity-expression-for-space-charge-limited-conduction-inferroelectrics-and-other-so

\section{INTECH}

open science | open minds

\author{
InTech Europe \\ University Campus STeP Ri \\ Slavka Krautzeka 83/A \\ 51000 Rijeka, Croatia \\ Phone: +385 (51) 770447 \\ Fax: +385 (51) 686166 \\ www.intechopen.com
}

\author{
InTech China \\ Unit 405, Office Block, Hotel Equatorial Shanghai \\ No.65, Yan An Road (West), Shanghai, 200040, China \\ 中国上海市延安西路65号上海国际贵都大饭店办公楼 405 单元 \\ Phone: +86-21-62489820 \\ Fax: +86-21-62489821
}


(C) 2011 The Author(s). Licensee IntechOpen. This chapter is distributed under the terms of the Creative Commons Attribution-NonCommercialShareAlike-3.0 License, which permits use, distribution and reproduction for non-commercial purposes, provided the original is properly cited and derivative works building on this content are distributed under the same license. 